\title{
Changes in the Biochemical Parameters of Rat Blood when Administering Toxic Doses of Sodium Valproate
}

\author{
Lyudmila V. Okhremchuk, $\mathrm{PhD}^{1}$; Igor Zh. Seminskiy, $\mathrm{PhD}, \mathrm{ScD}^{1}$; \\ Marina A. Darenskaya, PhD, $\mathrm{ScD}^{2 *}$; Lyubov I. Kolesnikova, Academician of the RAS ${ }^{2}$; \\ Sergey I. Kolesnikov, Academician of the RAS ${ }^{2}$ \\ IIrkutsk State Medical University \\ ${ }^{2}$ Scientific Centre for Family Health and Human Reproduction Problems \\ Irkutsk, the Russian Federation
}

\begin{abstract}
Background: The aim of this research was to study the regularities of changes in blood biochemical parameters in outbred rats exposed to the toxic effect of sodium valproate (SV) in the experiment.

Methods and Results: The study was performed on 94 white outbred male rats, weighing 190-210g. According to the research protocol, all animals were divided into two groups: Intact Group (IG, n=30) and Experimental Group (EG, n=64). Animals of EG were given the anticonvulsant SV in a toxic dose of $600 \mathrm{mg} / \mathrm{kg}$, intragastrically, once a day, for $7(\mathrm{n}=23), 14(\mathrm{n}=13), 21(\mathrm{n}=13)$ and $28(n=15)$ days. The animals were withdrawn from the experiment after 7, 14, 21 and 28 days using diethyl ether. The following biochemical parameters in blood serum were determined: AST (aspartate aminotransferase), ALT (alanine aminotransferase), ALP (alkaline phosphatase), GGT ( $\gamma$-glutamyltransferase), TP (total protein), glucose, direct bilirubin, TC (total cholesterol), and $\mathrm{TG}$ (triglycerides). The administration of toxic doses of SV to experimental animals was accompanied by changes in the biochemical status of blood, especially pronounced on Days 21 and 28 of the experiment. In particular, the activity of enzymes AST, ALT, ALP, GGT increases, the levels of TP and glucose decrease, and the levels of direct bilirubin, TG and TC, increase.

Conclusion: The obtained data showed that the overdose of SV significantly worsens the blood biochemical parameters, which may indicate a toxic damage of hepatocytes. The studied biochemical parameters should be used to assess late effects of anticonvulsants on the body. (International Journal of Biomedicine. 2020;10(3):266-269.)
\end{abstract}

Key Words: rats $\bullet$ sodium valproate $\bullet$ toxic dose $\bullet$ biochemical parameters $\bullet$ blood serum

\section{Abbreviations}

ALP, alkaline phosphatase; ALT, alanine aminotransferase; AST, aspartate aminotransferase; GGT, $\gamma$-glutamyltransferase; SV, sodium valproate; TC, total cholesterol; TG, triglycerides; TP, total protein.

\section{Introduction}

Epilepsy refers to common neurological diseases associated with high mortality, stigmatization and economic costs. ${ }^{(1)}$ The prevalence of this pathological condition in the

*Corresponding author: Marina A. Darenskaya, PhD, ScD. Scientific Centre for Family Health and Human Reproduction Problems, Irkutsk, the Russian Federation. E-mail: marina darenskaya@inbox.ru world is 6.38 per 1000 population, in Russia about 3.22 per 1000 population. ${ }^{(2)}$ Epilepsy is characterized by occasional epileptic attacks resulting from excessive neuronal discharges, accompanied by a variety of clinical and paraclinical symptoms. ${ }^{(3)}$ The main method of epilepsy treatment is pharmacotherapy with anticonvulsants along with lifestyle changes targeted at preventing attacks and related complications. ${ }^{(1,3)}$

Currently, a preparation of sodium valproate (SV) is widely used in the treatment of patients with epilepsy. ${ }^{(4)}$ The main effect of SV is aimed at preventing the development of 
epileptic attacks and cognitive deficits, which is achieved by long-term drug therapy, on average from two to five years or more, or lifelong. ${ }^{(1,5)}$ Many metabolites of SV are biologically active substances, which, on the one hand, can lead to a potential clinical effect and, on the other hand, can lead to chronic intoxication of the organism. ${ }^{(4-9)}$ It was found that SV in high doses has a pronounced neurotoxic effect with the development of cognitive disorders, increased anxiety, etc. ${ }^{(6)}$ The frequency of side effects during anticonvulsant therapy varies from $7 \%$ to $25 \%$, in some cases it can reach $68.3 \%$. ${ }^{(4)}$ An additional risk factor for the development of side effects is failure to follow instructions, which may concern indications, contraindications, and dosages when prescribing drugs, their co-use with other drugs, age of patients, etc. ${ }^{(6,7)}$

Due to long-term, often life-long therapy, as well as numerous negative effects, the drugs of this group have special requirements for their safety and impact on patients' quality of life. ${ }^{(10)}$ It is known that most of the basic anticonvulsants, also SV, are metabolized by the generation of reactive metabolites. They can covalently bind macromolecules, which results not only in the suppression of epileptic attacks, but also in the development of systemic toxicity, especially hepatotoxicity. ${ }^{(11-13)}$

Thereby, the aim of this research was to study the regularities of changes in blood biochemical parameters in outbred rats exposed to the toxic effect of sodium valproate in the experiment.

\section{Materials and Methods}

The study was performed on 94 white outbred male rats, weighing 190-210 g. The animals were kept in standard plastic cages in natural light, with free access to water and food (standard food in an amount corresponding to the daily norms). The experiment was conducted on the basis of a certified vivarium of the "East-Siberian Institute for Medical and Environmental Studies."

According to the research protocol, all animals were divided into two groups: Intact Group (IG, $n=30$ ) and Experimental Group (EG, $\mathrm{n}=64$ ). Animals of $\mathrm{EG}$ were given the anticonvulsant $\mathrm{SV}$ in a toxic dose of $600 \mathrm{mg} / \mathrm{kg}$, intragastrically, once a day, for $7(n=23), 14(n=13), 21(n=13)$ and $28(\mathrm{n}=15)$ days. The dosage of SV was calculated based on the average daily dose for a person weighing $60 \mathrm{~kg}$, which is $1200-2100 \mathrm{mg}$ per day. The dose was increased by $50 \%$ relative to animal body weight. ${ }^{(5)}$ The animals were withdrawn from the experiment after 7, 14, 21 and 28 days using diethyl ether. Blood was drawn into dry sterile tubes on an empty stomach at the same time. To obtain serum, the blood was centrifuged for $15 \mathrm{~min}$ at $3000 \mathrm{rpm}$. The following biochemical parameters in blood serum were determined: AST, ALT, ALP, GGT, TP, glucose, direct bilirubin, TC, and TG..$^{(14)}$

Work on the animals was done in compliance with the principles of the Helsinki Declaration on the humane treatment of animals, stated in normative documents of the European community(86/609/EU), Manual on Experimental (Preclinical) Study of New Pharmacological Substances, and "Good laboratory practice" (MHRF Order No. 708H dated 23.08.2010).
Statistical analysis was performed using the Statistica 6.1 software package (Stat-Soft Inc., USA). The normality of distribution of continuous variables was tested by the Kolmogorov-Smirnov test with the Lilliefors correction and Shapiro-Wilk test. For descriptive analysis, results are presented as mean \pm standard deviation (SD), median (Me), interquartile range (IQR; 25 th to 75 th percentiles). For data with normal distribution, inter-group comparisons were performed using Student's t-test. Differences of continuous variables departing from the normal distribution, even after transformation, were tested by the Mann-Whitney U-test. A probability value of $P \leq 0.05$ was considered statistically significant.

\section{Results and Discussion}

The results of the research showed that with daily administration of SV in EG, the AST activity statistically significantly increased on Days 7 and 14 of the experiment (by 1.61 times $(P=0.0187)$ and 1.28 times $(P=0.0408)$, respectively), compared to IG (Fig.1). Simultaneous increase in the values of AST and ALT was recorded on Day 21 (by 3.67 times $(P=0.0118)$ and 2.33 times $(P=0.0221)$, respectively $)$, as well as on Day 28 (by 3.67 times $(P=0.0147)$ and 2.33 times $(P=0.019)$, respectively). Similarly, the serum activity of ALP and GGT was changed (Fig.1). Thus, the ALP activity increased on Days 7 and 14 (by 1.56 times $(P=0.0405)$ and 1.54 times $(P=0.0141)$, respectively). On Days 21 and 28 of the experiment, there was a statistically significant increase in the activity of ALP and GGT in relation to the data of intact rats: the ALP level increased by 3.58 times $(P=0.0261)$ and 3.59 times $(P=0.0321)$, and the GGT level increased by 3 times $(P=0.0034)$ and 3.79 times $(P=0.0247)$, respectively.

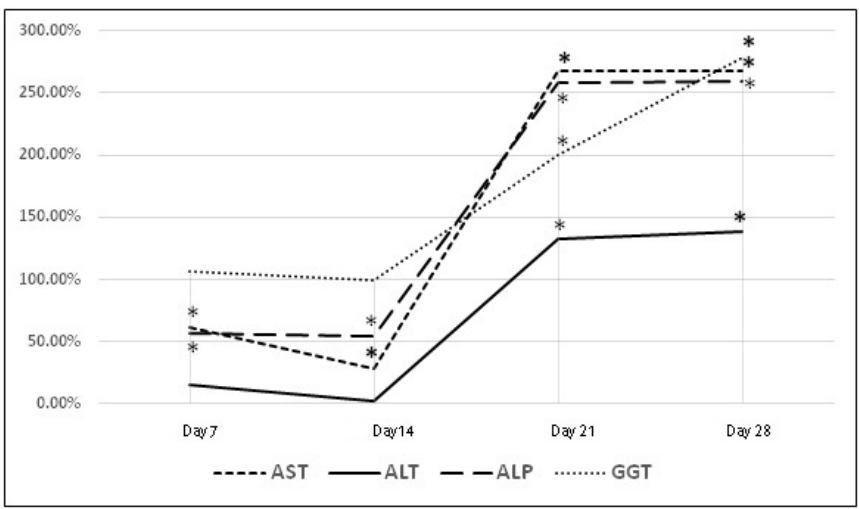

Fig. 1. Dynamics of enzyme activity changes in rats when administered toxic doses of SV: * - statistically significant differences with control values.

An overdose of SV led to a moderate decrease in the serum levels of TP and glucose (Fig.2). On Day 21, the levels of TP and glucose decreased by 1.25 times $(P=0.0124)$ and 1.41 times $(P=0.0155)$, respectively. On Day 28 , the fall in values of TP and glucose was more intensive: by 1.49 times $(P=0.0306)$ and 1.81 times $(\mathrm{P}=0.0322)$, respectively The level of direct bilirubin increased on Days 21 and 28 (by 2.5 times ( $P=0.0141)$ and 2.92 times $(P=0.0225)$, respectively) (Fig. 2$)$. 
The TC content increased gradually by 1.65 times $(P=0.0459)$ on Day 7 and more than 3 times on Days $21(P=0.0244)$ and $28(P=0.0063)$. The concentration of TG also increased on Day 21 (by 1.54 times; $P=0.0193$ ), reaching its maximum on Day 28 (by 1.73 times; $P=0.0312$ ).

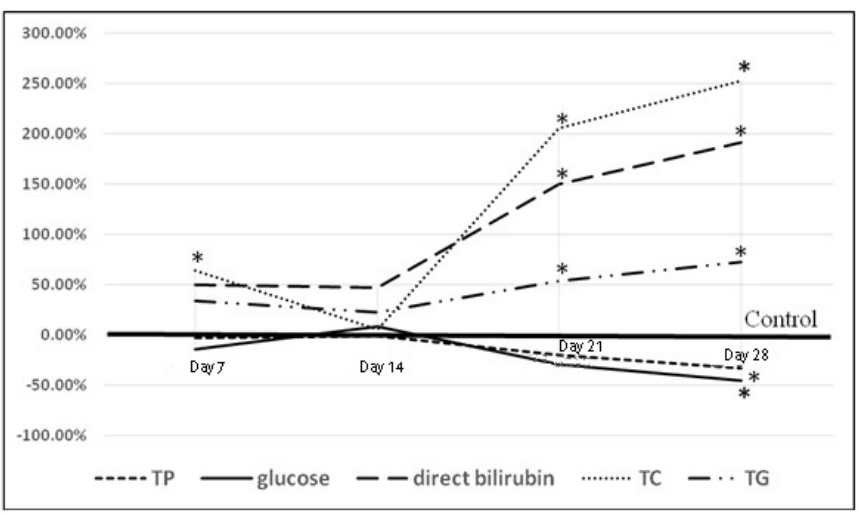

Fig. 2. Dynamics of changes in blood biochemical parameters in rats when administered toxic doses of $\mathrm{SV}$;

* - statistically significant differences with control values.

As shown by the obtained data, when toxic doses of SV were administered, there was an increase in the activity of liver enzymes AST and ALT, with a maximum on Days 21 and 28 of the experiment. These changes are common for cytolysis, the main syndrome of pathological liver damage. Intensive release of these enzymes from damaged hepatocytes indicates hepatocellular damage, and, in addition, may be associated with cholestatic reactions, when bile backflow is toxic to liver cells. ${ }^{(11)}$ Liver metabolites of SV are known to be powerful alkylating, arylating and acetylating agents, which covalently combine with hepatocytic macromolecules. ${ }^{(15)}$ Free-radical reactions are activated, with a logical decrease in the activity of the antioxidant defense system. ${ }^{(16-23)} \mathrm{In}$ particular, there is expressed insufficiency of glutathione, a very important antioxidant. It also acts as a cofactor of a number of enzymes involved in detoxification of xenobiotics. (16,24) In a toxic lesion, the mitochondrial apparatus of cells suffers damage and dysfunction. It has been established that the metabolite of SV (2-propylpentanic acid) is able to directly block enzymes of the mitochondrion respiratory chain and actively participate in suppression of $\beta$-oxidation of fatty acids. ${ }^{(10,11)}$ Inhibition of $\mathrm{K}^{+} / \mathrm{Na}^{+} \mathrm{ATPase}$, glycolysis depression, separation of oxidative phosphorylation, and disruption of the ways in which an excess of fatty acids is utilized are observed. ${ }^{(25)}$ These processes have an extremely negative impact on the condition of liver cells, and in the future may lead to the development of chronic liver dystrophy, the socalled fatty hepatosis. Damage of mitochondria in hepatocytes is manifested by an increased mitochondrial apparatus with its significant swelling, reduction in the number of mitochondria per field of view, and specific paracrystalline inclusions in the mitochondrial matrix with reduced density. ${ }^{(6,7)}$

Damage to hepatocytes may contribute to disturbance of the synthesis, secretion or transport of bile and lead to clinical manifestations of cholestasis. ${ }^{(2)}$ Severe SV intoxication will contribute to significant damage to the structure of liver cells, their necrotic changes, resulting in an increase in plasma transaminases, ALP, GGT, as well as bilirubin, especially expressed in the second period of the research. The increased values of ALP may indicate the presence of structural and functional disorders of hepatocyte membranes, which is typical for infiltrative liver diseases. ${ }^{(6)}$ Decrease in the level of TP may indicate low ability of the liver to metabolize amino acids, as a result of which a number of metabolic reactions may be impaired. Our data are consistent with results indicating that high doses of SV ( $80 \mathrm{mg} / 100 \mathrm{~g}$ of body weight) significantly reduce protein synthesis by liver cells. ${ }^{(1)}$ In addition, our study recorded increased concentrations of TC and TG, also on Days 21 and 28 of the experiment. In conditions of toxic effects of SV, it may indicate the development of lipid metabolism disorders.

Thus, the administration of toxic doses of SV to experimental animals is accompanied by changes in the biochemical status of blood, especially pronounced on Days 21 and 28 of the experiment. In particular, the activity of enzymes AST, ALT, ALP, GGT increases, the levels of TP and glucose decrease, and the levels of direct bilirubin, TG and TC, increase, which may indicate a toxic damage of hepatocytes. The studied parameters should be used to assess late effects of anticonvulsants on the body.

\section{Competing Interests} interests.

The authors declare that they have no competing

\section{References}

1. Kobow K, Blümcke I. Epigenetics in epilepsy. Neurosci Lett. 2018;667:40-46. doi:10.1016/j.neulet.2017.01.012

2. Fiest KM, Sauro KM, Wiebe S, Patten SB, Kwon $\mathrm{ChS}$, Dykeman J, et al. Prevalence and incidence of epilepsy: A systematic review and meta-analysis of international studies [published correction appears in Neurology. 2017 Aug 8;89(6):642]. Neurology.2017;88(3):296-303. doi:10.1212/ WNL.0000000000003509

3. Iffland PH 2nd, Crino PB. The role of somatic mutational events in the pathogenesis of epilepsy. Curr Opin Neurol. 2019;32(2):191-197. doi:10.1097/WCO.0000000000000667

4. Li J, Yang D, Zhao D, Li N, Lin W. Efficacy of phenobarbital and sodium valproate in treating convulsive epilepsy in rural northeast China. Seizure. 2019;71:207-213. doi:10.1016/j.seizure.2019.06.012

5. Gusev EI. Epilepsy and its treatment. M.: GEOTARMedia. 2016. [Textbook in Russian].

6. Ivashkin VT, Baranovsky AYu, Raikhelson KL, Palgova LK, Maevskaya MV, Kondrashina EA, et al. Drug-Induced Liver Injuries (Clinical Guidelines for Physicians). Russian Journal of Gastroenterology, Hepatology, Coloproctology. 2019; 29(1):101-131. [Article in Russian].

7. Bunchorntavakul C, Reddy KR. Review article: herbal and dietary supplemen the patotoxicity. Aliment Pharmacol Ther. 2013;37(1):3-17. doi:10.1111/apt.12109

8. Kolesnikova LI, Darenskaya MA, Kolesnikov SI. Free radical oxidation: pathophysiologist's view. Bulletin of 
Siberian Medicine. 2017;16(4):16-29. [Article in Russian]. 9. Kolesnikova LI, Kolesnikov SI, Romanova ED, Chkhenkeli VA, Darenskaya MA, Grebenkina LA, et al. Effect of Preparation Based on Trametes Pubescens Xylotroph Fungi on Lipid Peroxidation in the Blood of Experimental Animals under Conditions of Dark Stress. Bull Exp Biol Med. 2017;162(6):762-764. doi:10.1007/s10517-017-3707-0

10. Xu S, Chen Y, Ma Y, Liu T, Zhao M, WangZ, etal. Lipidomic Profiling Reveals Disruption of Lipid Metabolism in Valproic Acid-Induced Hepatotoxicity. Front Pharmacol. 2019;10:819. Published 2019 Jul 19. doi:10.3389/fphar.2019.00819

11. Abdelkader NF, Elyamany M, Gad AM, Assaf N, Fawzy HM, Elesawy WH. Ellagic acid attenuates liver toxicity induced by valproic acid in rats. J Pharmacol Sci. 2020;143(1):23-29. doi:10.1016/j.jphs.2020.01.007

12. Fedotova IB, Perepelkina OV, Nikolaev GM, Surina NM, Poletaeva II. Effect of Ethosuximide on Audiogenic Epilepsy in Krushinsky-Molodkina Rats. Bull Exp Biol Med. 2019;167(4):464-466. doi:10.1007/s10517-019-04550-9

13. Darenskaya MA, Grebenkina LA, Sholokhov LF, Rashidova MA, Semenova NV, Kolesnikov S.I., et al. Lipid peroxidation activity in women with chronic viral hepatitis. Free Radical Biology \& Medicine. 2016;100(S):S192.

14. Karpishchenko AI. Medical laboratory technology: a guide to clinical laboratory diagnostics (Vol 2). M.:GEOTARmedia; 2013. [Textbook in Russian].

15. Mohi-Ud-Din R, Mir RH, Sawhney G, Da MA, Bhat ZA. Possible Pathways of Hepatotoxicity Caused by Chemical Agents. Curr Drug Metab. 2019;20(11):867-879. doi:10.217 4/1389200220666191105121653

16. Roganovic M, Pantovic S, Dizdarevic S. Role of the oxidativve stress in the pathogenesis of epilepsy. Neurol Sci Neurophysiol 2019; 36(1):1-8.

17. Darenskaya MA, Rychkova LV, Kolesnikov SI, Gavrilova OA, Kravtsova OV, Grebenkina LA, et al. Oxidative Stress Parameters in Adolescent Boys with Exogenous-Constitutional Obesity. Free Radical Biology and Medicine. 2017;112:129-130. 18. Kolesnikova LR, Darenskaya MA, Rychkova LV,
Pogodina AV, Grebenkina LA, Kolesnikov SI, et al. Oxidative stress parameters and state of regional periodontal blood flow in adolescents with arterial hypertension and periodontal diseases. International Journal of Biomedicine. 2018;8(4):301305. doi: 10.21103/Article8(4)_OA6

19. Kolesnikova LI, Rychkova LV, Kolesnikova LR, et al. Coupling of Lipoperoxidation Reactions with Changes in Arterial Blood Pressure in Hypertensive ISIAH Rats under Conditions of Chronic Stress. Bull Exp Biol Med. 2018;164(6):712-715. doi:10.1007/s10517-018-4064-3

20. Darenskaya MA, Kolesnikov SI, Rychkova LV, Grebenkina LA, Kolesnikova LI. Oxidative stress and antioxidant defense parameters in different diseases: ethnic aspects. Free Radical Biology \& Medicine. 2018;120(S1):60.

21. Kolesnikova LI, Kolesnikov SI, Darenskaya MA, Grebenkina LA, Nikitina OA, Lazareva LM, et al. Activity of LPO Processes in Women with Polycystic Ovarian Syndrome and Infertility. Bull Exp Biol Med. 2017;162(3):320-322. doi:10.1007/s10517-017-3605-5

22. Bairova TA, Kolesnikov SI, Kolesnikova LI, Pervushina OA, Darenskaya M.A, Grebenkina LA. Lipid peroxidation and mitochondrial superoxide dismutase-2 gene in adolescents with essential hypertension. Bull Exp Biol Med. 2014;158(2):181-184. doi:10.1007/s10517-014-2717-4

23. Darenskaya MA, Gavrilova OA, Rychkova LV, Kravtsova OV, Grebenkina LA, Osipova EV, et al. The assessment of oxidative stress intensity in adolescents with obesity by the integral index. International Journal of Biomedicine. 2018;8(1):37-41. doi:10.21103/Article8(1)_OA5

24. Kolesnikova LI, Kolesnikova LR, Darenskaya MA, Natyaganova LV, Grebenkina LA, Korytov LI, et al. Evaluation of Lipid Peroxidation-Antioxidant Defense System Depending on the Stage of Stress Reaction in Hypertensive ISIAH Rats. Bull Exp Biol Med. 2019;166(5):610-612. doi:10.1007/ s10517-019-04402-6

25. Neuman MG. Biomarkers of Drug-Induced Liver Toxicity. Ther Drug Monit. 2019;41(2):227-234. doi:10.1097/ FTD.0000000000000610 